\title{
Depth Data Improves Skin Lesion Segmentation
}

\author{
Xiang $\operatorname{Li}^{1}$, Ben Aldridge ${ }^{2}$, Lucia Ballerini ${ }^{1}$, Bob Fisher $^{1}$, and Jonathan Rees ${ }^{2}$ \\ 1 School of Informatics, University of Edinburgh, UK \\ x.li-29@sms.ed.ac.uk, lucia.ballerini@ed.ac.uk, rbf@inf.ed.ac.uk \\ 2 Dermatology, University of Edinburgh, UK \\ ben.aldridge@ed.ac.uk, jonathanlrees@mac.com
}

\begin{abstract}
This paper shows that adding 3D depth information to RGB colour images improves segmentation of pigmented and non-pigmented skin lesion. A regionbased active contour segmentation approach using a statistical model based on the level-set framework is presented. We consider what kinds of properties (e.g., colour, depth, texture) are most discriminative. The experiments show that our proposed method integrating chromatic and geometric information produces segmentation results for pigmented lesions close to dermatologists and more consistent and accurate results for non-pigmented lesions.
\end{abstract}

\section{Introduction}

Segmentation is the first step of computer-based skin lesion diagnosis and its importance is twofold. First, the lesion boundary provides important information for accurate diagnosis. Second, the extraction of other clinical features critically depends on the accuracy of the boundary [1]. Due to reasons such as low contrast between the lesion and its background, artifact inference, etc., segmentation is a very challenging task. In recent years, many methods have been proposed for lesion boundary detection. Classic algorithms such as histogram thresholding, region-growing, k-means are widely used to segment lesions into homogeneous regions based on their intensity values. Xu et al. [2] introduced a semi-automatric method based on thresholding. Experiment results showed an average error that was about the same as that obtained by experts. Iyatomi et al. [3] proposed a dermatologist-like lesion region extraction algorithm that combined both pixel-based and region-based methods and introduced a regiongrowing approach which aimed to bring the extraction results closer to those determined by dermatologists. More recently, optimization based segmentation methods, especially active contours, have been applied to segment lesion images and have become more popular as they can produce decent results $[4,5]$. Tang presented a skin cancer segmentation algorithm using a multi-directional gradient vector flow snake [6]. The performance of their algorithm is close to human segmentation. Yuan et al. [7] proposed a novel multi-modal skin lesion segmentation method based on region fusion and narrow band energy graph 
partitioning. Comparisons showed that their method outperformed the state of the art methods with a mean error rate of $12.41 \%$ for $X L M$ (oil immersion and cross-polarizaion mode of epiluminescence microscopy $(E L M))$ images and $12.28 \%$ for $T L M$ (side-transillumination mode of $E L M$ ) images. They only used intensity features and an extension to incorporate colour and texture features was considered as future work. Unfortunately, most of these methods are developed for dermoscopy images and focus on pigmented melanocytic lesions (e.g., distinguishing melanoma from benign naevi). They are not suitable for the nonpigmented lesions, including two other important skin cancers BCC (Basal Cell Carcinoma) and SCC (Squamous Cell Carcinoma) for which early and correct diagnosis is also of great importance. They are included in our work.

In this paper, we present a region-based active contour segmentation approach and apply it to both pigmented and non-pigmented lesion data including 2D conventional colour data and 3D topological (depth) data, which correlates strongly with human visual assessment of lesion surface appearance. The skin lesion data in this research is collected with a non-contact instantaneous dense stereophotometry system (equipped with ring flash for consistent lighting and a Macbeth colour chart), which outputs both dense 3D point cloud data and 1:1 aligned colour images [8]. An example of the image data is shown in Fig. 1 and Fig. 2(e)

For the level-set formulation of active contour segmentation, partitioning a given image is achieved by minimizing appropriate energy functions. Partial differential equations are used to drive the contours, which are implicitly represented as the (zero) level line of some embedding function, to evolve in the direction of a negative energy gradient [9]. Chan et al. proposed a region-based segmentation model using the Mumford-Shah functional [10]. Our method is inspired by another region-based level set segmentation using Bayesian inference [9]. It partitions the image domain by progressively fitting statistical models to the properties in each of a set of regions. The probabilistic formulation of the segmentation problem considers segmentation as a process of finding an optimal partition $P(\Omega)$ of the image domain by maximizing the a posteriori probability $p(P(\Omega) \mid I)$ for a given image $I$, integrating a regularity constraint.

\section{Method}

For active contour segmentation, an initial contour is needed as a first step of segmentation. We use a semi-automatic strategy, in which the initial contour is drawn roughly by hand. The level-set segmentation framework is used to refine this initial contour according to the regional information of the lesions.

For binary segmentation, the level-set formulation [9] is expressed as

$$
E(\phi)=\int_{\phi \in \Omega}-H(\phi) \log p_{1}(f)-(1-H(\phi)) \log p_{2}(f)+\nu|\nabla H(\phi)| d \phi .
$$

$H(\phi)$ denotes the heaviside step function, $p_{1} / p_{2}$ are the pdfs inside/outside the contour. The first two terms in (1) model the areas inside and outside the contour 
while the last term represents the length of the separating contour. Considering the associated Euler-Lagrange equation for $\phi$, the minimization of the energy functional by a gradient descent of the embedding function $\phi$ is [11]:

$$
\frac{\partial \phi}{\partial t}=-\frac{\partial E(\phi)}{\partial \phi}=\delta(\phi)\left(\nu \operatorname{div}\left(\frac{\nabla \phi}{|\nabla \phi|}\right)+\log \frac{p_{2}(f(x))}{p_{1}(f(x))}\right) .
$$

$\delta(\phi)$ has value 1 at the lesion boundary and 0 elsewhere.

In the following, the two questions concerning the above function are addressed: 1) how to chose a probabilistic model to fit the density distribution of properties and 2) which features or properties $f(x)$ should be used.

\subsection{Distributions}

Parametric density functions $p(f(x) \mid \theta)$ are used to model distributions. For a particular choice of parametric density, parameters $\theta$ modeling the distribution depend on the associated regions and update with the evolution of the contour.

Gaussian mixture model extension Lesion regions usually do not have a homogeneous content, especially for BCC and SCC (this is also the case for the background normal skin region because of hairs and skin markings). Hence, the density distribution of a property may have multiple peaks. This implies that the commonly used single multivariate Gaussian or Poisson model might not fit the data well. A multivariate Gaussian mixture model developed using an expectation-maximization(EM) algorithm was the final selected representation, shown in Fig. 1. The initial cluster parameters of components are determined by $k$-means algorithm. The number of the clusters are determined by optimization which chooses the largest average silhouette of the data (typically $K=2$ to 3 for lesion region and $K=1$ to 2 for skin region). The final evolution equation is

$$
\frac{\partial \phi}{\partial t}=\delta(\phi)\left(\nu \operatorname{div}\left(\frac{\nabla \phi}{|\nabla \phi|}\right)+\log \frac{p_{\text {mixSkin }}\left(f(x) \mid \mu_{1}, \Sigma_{1}, \ldots, \mu_{K}, \Sigma_{K}\right)}{p_{\text {mixLesion }}\left(f(x) \mid \mu_{1}, \Sigma_{1}, \ldots, \mu_{K}, \Sigma_{K}\right)}\right) .
$$

\subsection{Image properties}

One central question is which properties characterize lesions and distinguish them from the background skin?

Colour Colour is the most direct and critical property for dermatologists to assess and diagnose skin lesions, but which colour space or colour elements should be used? Here, the colour representation of lesions combines the results from different channels of different colour spaces. It includes 1) the Saturation of $H S V, 2) a *$ of $\left.C I E_{-} L a b, 3\right)$ the normalized blue of $R G B$ as lesions are often more prominent in this channel [1] and 4) the Hue of $H S V$. Hence, each image position is associated with a colour-valued feature vector, as $f(x)=\left(I_{\text {saturation }}, I_{a *}, I_{\text {blue }}, I_{\text {hue }}\right)^{T}$. As shown in Fig. 2, the lesion area, especially the right part which is similar to surrounding skin is enhanced compared with the conventional $R G B$ representation. 
Relative depth Lesion surface appearance attributes can be grouped into two major categories - chromatic and geometric attributes. The former has been extensively used. Little research has been done on geometric (or depth) properties to lesion segmentation. Our stereo imaging system obtains depth information as well as colour. We extract the relative depth $I_{\text {depth }}$ between the current pixel and a quadric surface fitted to the background [8] to account for local surface shape, shown in Fig. 2(e). The texture of the depth data is also used.

Texture Texture is an important property for lesion diagnosis, since it differs among different lesion types, as well as different locations of skin (e.g., lesion and healthy skin). We assign a local texture signature to each image location. A well known local representation is the gradient structure tensor which has good properties for texture discrimination and is widely used to represent texture [9]. It is a matrix of first partial derivatives. For an intensity image, the structure tensor is expressed as $J=\left(\begin{array}{cc}I_{x 1}^{2} & I_{x 1} I_{x 2} \\ I_{x 1} I_{x 2} & I_{x 2}^{2}\end{array}\right)$. The associated texture properties at each image location can be represented as $f(x)=\left(J_{1}, J_{2}, J_{3}\right)=\left(\frac{I_{x 1}^{2}}{|\nabla I|}, \frac{2 I_{x 1} I_{x 2}}{|\nabla I|}, \frac{I_{x 2}^{2}}{|\nabla I|}\right)^{T}$. The first derivatives $\left(I_{x 1}\right.$ and $\left.I_{x 2}\right)$ of an image are not rotationally invariant. To compensate, we adopted the steerable Gaussian filter proposed in [12] to calculate the directional derivative $I_{x 1}$ oriented at angle $\alpha$ with respect to the $x$-axis and $I_{x 2}$ at degree $\alpha+90^{\circ} . \alpha$ starts at $0^{\circ}$ degrees and increases by $15^{\circ}$ until $90^{\circ}$. The texture property at each image location is the average. Next, we sum the tensors of the individual channels. An adaptive anisotropic diffusion method is applied to smooth homogeneous regions while inhibiting diffusion in highly textured regions: $\frac{\partial J}{\partial t}=\operatorname{div}[c(|\nabla J|) \nabla J], J(t=0)=J_{0}$.

We adopted the diffusion conductance proposed by Perona and Malik [13] as $c(x)=\exp \left(-\frac{x^{2}}{P^{2}}\right) \cdot c$ varies as a function of the texture properties. It is small where the gradient of the property image is large, resulting in lower diffusion near the textured locations like boundaries [14]. Two modifications are applied to improve the performance of the diffusion filter. First, the property image is smoothed by a Gaussian filter with parameter $\sigma$ decreasing at each iterations. Second, we compute $P$ adaptively as a function of time - higher at beginning and lower gradually. The time duration for the evolution of the diffusion function is determined experimentally as 20 iterations. The diffused structure tensor images are given in Fig. 2 (f), (g), (h). The textural difference can be seen between the lesion and its surrounding skin. The final property vector with colour, depth and texture properties is

$$
f(x)=\left(I_{\text {saturation }}, I_{a *}, I_{\text {blue }}, I_{\text {hue }}, I_{\text {depth }}, \sum_{i=1}^{M} \frac{I_{i x 1}^{2}}{\left|\nabla I_{i}\right|}, \sum_{i=1}^{M} \frac{I_{i x 1} I_{i x 2}}{\left|\nabla I_{i}\right|}, \sum_{i=1}^{M} \frac{I_{i x 2}^{2}}{\left|\nabla I_{i}\right|}\right)^{T},
$$

where $M$ is the number of colour and depth images. Here, $\mathrm{M}=5$.

\section{Results}

To reduce the influence of artifacts like hair and intrinsic cutaneous features (e.g., blood vessels, skin lines), image smoothing using $5 \times 5$ mean filtering is 


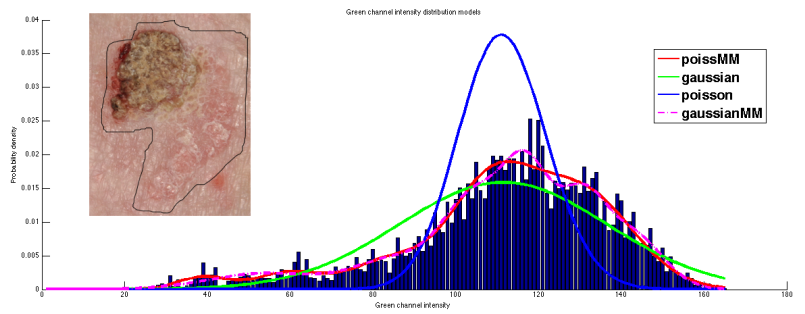

Fig. 1. The univariate density models of the lesion region on the green channel. The top-left shows the corresponding lesion - a SCC with dried exudate (The lesion region is identified with the black curve).

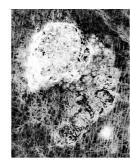

(a)

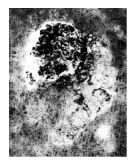

(b)

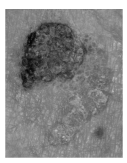

(c)

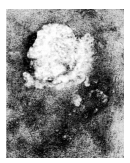

(d)

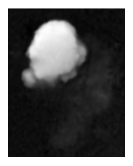

(e)

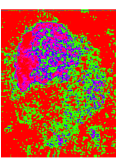

(f)

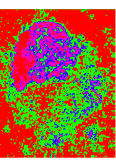

(g)

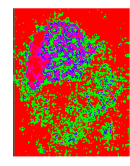

(h)

Fig. 2. Colour properties of the lesion in Fig 1. (a) Saturation, (b) $a *$, (c) Blue, (d) Hue, (e) Stretched depth, (f)-(h)colour coded tensor elements $\frac{I_{x 1}^{2}}{|\nabla I|}, \frac{2 I_{x 1} I_{x 2}}{|\nabla I|}$ and $\frac{I_{x 2}^{2}}{|\nabla I|}$.

applied. After segmentation, post processing included hole filling, small segment deletion and local region growing.

The 20 test images used in our comparison are randomly selected from our lesion data-base, including 2 SCC, 4 ML (Melanocytic nevus), 7 BCC, 1 AK (Actinic Keratosis) and 6 SK (Seborrhoeic Keratosis). Seven of them are pigmented lesions; while the other 13 are non-pigmented. These images are shown in Fig. 3 along with our segmentation results and corresponding lesion types. Manual segmentations given by 8 dermatologists from the Dermatology department of Edinburgh University are used for performance evaluation. A standard tumour area (STA) is defined as the region selected by four or more dermatologists.

The standard deviation (SD) of the area of manual segmentations is calculated for each lesion image and the value is normalized by the corresponding STA. There are big variations between dermatologists. The average SD over our 20 test images is $20.69 \%$. There is more variation in clinical opinion of lesion boundaries for non-pigmented lesions $(\mathbf{S D}=24.09 \%)$ than pigmented ones $(\mathbf{S D}=9.74 \%)$.

To evaluate computer-based segmentations, we used the popular segmentation evaluation criteria $X O R$ measure (or Error rate) defined as:

$$
X O R=\frac{\operatorname{Area}(A B \oplus M B)}{\operatorname{Area}(A B+M B)} \times 100 \%
$$



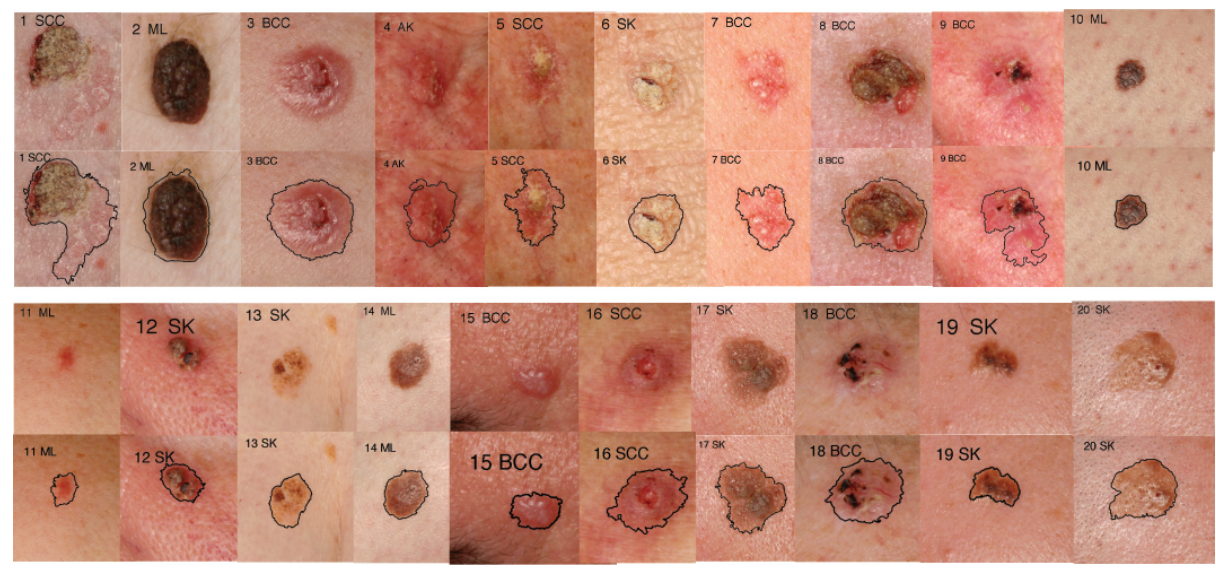

Fig. 3. The 20 test images and our segmentation results.

where $A B$ and $M B$ are the binary images obtained by computer and the reference segmentation (STA), respectively. $\bigoplus$ denotes exclusive-OR and gives the pixels for which $A B$ and $M B$ disagree; + means union.

Based on this quantitative metric, we performed a comparison study of our method using different properties and the results are summarized as:

1. Our colour combination performed the best (average error rate of 11.14\%) compared to the commonly used CIE_Lab colour space (11.71\%).

2. Integrating colour and depth information reduces the error rate from $11.14 \%$ to $10.74 \%$. Similarly, the colour structure tensor results are reduced from $10.80 \%$ to $9.68 \%$ by being extended with depth as shown in Fig. 4 .

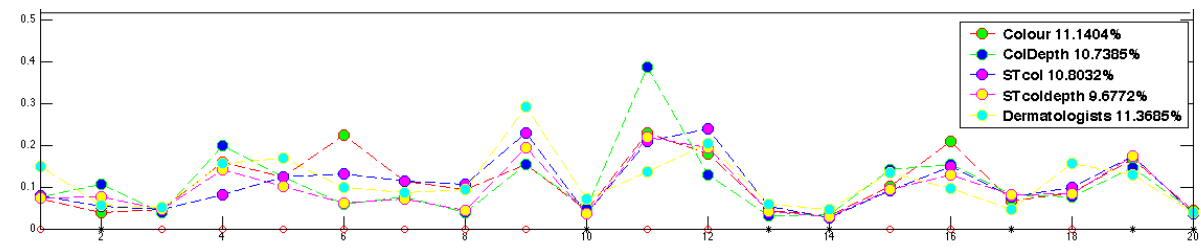

Fig. 4. Segmentation comparison. The associated lesions are shown in Fig. 3. Red - identifies non-pigmented lesions, black $*$ identifies pigmented lesions. Colour uses components 1-4 of $f(x)$, ColDepth uses 1-5, STcol uses 1-4, 6-8, STcoldepth uses 1-8.

We compared our method to both manual segmentations and a popular segmentation method [10] using the Mumford-Shah functional (MS method). The results are summarized in Table 1 . The error rate of individual dermatologists and its variation is calculated. Our method produces very close segmentation to the dermatologists on pigmented lesions and more consistent segmentations on most non-pigmented lesions. This improvement can be explained as the integration of depth information which cannot be visualized by dermatologists from $2 D$ colour images as well as complicated texture associated with depth information. 
Fig. 5 shows contours obtained using different segmentation methods of several non-pigmented lesions (e.g., case 6 , where the error rate improves significantly by integration depth and texture information, see Fig. 4).

\begin{tabular}{|c|c|c|c|}
\hline Error Rate \%(XOR) & Dermatologists & MS [10] & Our method \\
\hline Overall & $11.37(8.22)$ & $15.42(8.67)$ & $9.68(5.90)$ \\
\hline Pigmented & $6.39(3.09)$ & $10.46(7.9)$ & $6.8(5.08)$ \\
\hline Non-pigmented & $14.05(6.13)$ & $18.10(8.11)$ & $11.23(5.89)$ \\
\hline
\end{tabular}

Table 1 Average segmentation error rates and their standard deviations

It is hard to directly compare our approach to other algorithms because most other algorithms are designed for 1) other input modalities (e.g., dermoscopy) and 2) only melanocytic lesions. We did evaluate the Skin Cancer Segmentation software package [2], which is based on colour differences between the lesion and the surrounding skin and the thresholding algorithm (here called the DT method). Of our 20 test images, 12 failed totally, because the lesions did not have significant pigmentation. We only compared the segmentation performance on the remaining 8. Some results are shown in Fig. 5. (We tried to obtain the best performance by tuning of the DT method's 6 parameters.) Over the 8 usable lesions, our method provides a smaller average error rate ( $5.50 \%$ versus $12.46 \%)$ and for some cases, the error rate difference is significant $(7.43 \%$ versus $34.75 \%$ in Fig. 5 (a)).

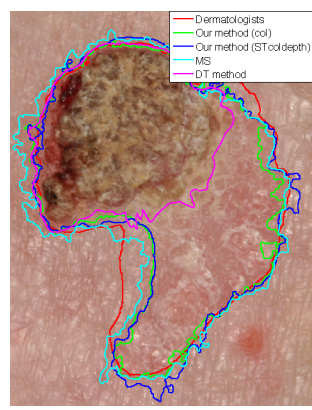

(a)

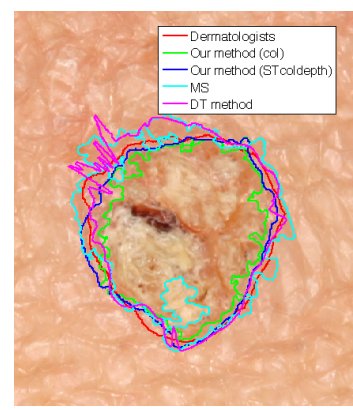

(b)

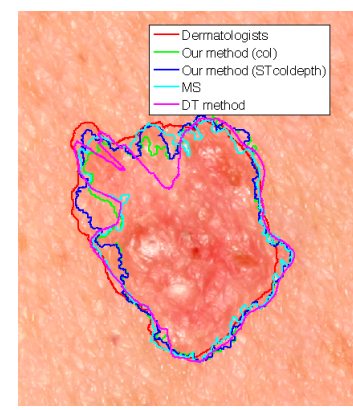

(c)

Fig. 5. The contours (cases 1, 6, 7 in Fig. 4) obtained by the dermatologists, $M S$ [10], DT [2] methods and our methods based on colour (col) or colour and depth structure tensor (STcoldepth) properties.

\section{Conclusion and further work}

A region-based probabilistic segmentation formulation using a statistical model within the level-set framework is applied to isolate lesions from their background. A multivariate gaussian mixture model is considered to be the best way to model the density distribution of properties. Upon comparison, we conclude that both depth and texture properties help to improve the segmentation result by reducing error rate from $15.42 \% \pm 8.67 \%$ to $9.68 \% \pm 5.90 \%$. Our method integrating colour, 
depth and texture information produces the best results compared to those by the MS method [10], the DT method [2] and dermatologists.

There are several potential improvements and follow-up work to be considered: 1) In addition to the structure tensor, other texture features should be considered. 2) Better colour representation of lesion images is needed as well as better preprocessing to reduce the influence of artifacts such as specular reflections. 3) We have not implemented weight selection between different kinds of properties (i.e., colour, depth and texture) and just treat them equally.

\section{References}

1. Celebi, M.E., Iyatomi, H., Schaefer, G., Stoecker, W.V.: Lesion border detection in dermoscopy images. Comp. Medical Imaging and Graphics 33 (2009) 148-153

2. Xu, L., Jackowski, M., Goshtasby, A., Roseman, D., Bines, S., Yu, C., Dhawan, A., Huntley, A.: Segmentation of skin cancer images. Image and Vision Computing 17(1) (1999) 65-74

3. Iyatomi, H., Oka, H., Saito, M., Miyaka, A., Kimoto, M., Yamagami, J.: Quantitative assessment of tumour area extraction from dermoscopy images and evaluation of the computer-based methods for automatic melanoma diagnostic system. Melanoma Research 16(2) (2006) 183-190

4. Chung, D.H., Sapiro, G.: Segmenting skin lesions with partial-differentialequations-bassed image processing algorithms. IEEE Transactions on Medical Imaging 19(7) (2000) 763-767

5. Erkol, B., Moss, R., Stanley, R., Stoecker, W., Hvatum, E.: Automatic lesion boundary detection in dermoscopy images using gradient vector flow snakes. Skin Research and Technology 11(17-26) (2005)

6. Tang, J.: A multi-direction GVF snake for the segmentation of skin cancer images. Pattern Recognition 42 (2009) 1172-1179

7. Yuan, X., Situ, N., Zouridakis, G.: A narrow band graph partitioning method for skin lesion segmentation. Pattern Recognition 42 (2009) 1017-1028

8. McDonagh, S.: Skin cancer surface shape based classification. Thesis, School of Informatics, University of Edinburgh (2008)

9. Cremers, D., Rousson, M., Deriche, R.: Reviews of statistical approaches to level set segmentation: integrating color, texture, motion and shape. International Journal of Computer Vision 72(2) (2007) 195-215

10. Chan, T.F., Vese., L.A.: Active contours without edges. IEEE Transactions in Image Processing 10(2) (2001) 266-277

11. Bertelli, L., Sumengen, B., Manjunath, B.S., Gibou, F.: A variational framework for multiregion pairwise-similarity-based image segmentation. IEEE Transactions on Pattern Analysis and Machine Intelligence 30(8) (2008) 1400-1415

12. Freeman, W.T., Adelson, E.H.: The design and use of steerable filters. IEEE Transactions on Pattern Analysis and Machine Intelligence 13(9) (1991) 891-906

13. Perona, P., Malik, J.: Scale-space and edge detection using anisotropic diffusion. IEEE Trans. on Pattern Analysis and Machine Intell. 12(7) (1990) 629-639

14. Weeratunga, S.K., Kamath, C.: Pde-based non-linear diffusion techniques for denoising scientific and industrial images: an empirical study. Image Processing: Algorithms and Systems Conference, SPIE 4667 (2002) 279-290 